Research Article

\title{
Elastic Equilibrium of Porous Cosserat Media with Double Porosity
}

\author{
Roman Janjgava \\ Ilia Vekua Institute of Applied Mathematics of Ivane Javakhishvili Tbilisi State University, 2 University Street, 0186 Tbilisi, Georgia \\ Correspondence should be addressed to Roman Janjgava; roman.janjgava@gmail.com
}

Received 11 May 2016; Accepted 30 June 2016

Academic Editor: John D. Clayton

Copyright (C) 2016 Roman Janjgava. This is an open access article distributed under the Creative Commons Attribution License, which permits unrestricted use, distribution, and reproduction in any medium, provided the original work is properly cited.

\begin{abstract}
The static equilibrium of porous elastic materials with double porosity is considered in the case of an elastic Cosserat medium. The corresponding three-dimensional system of differential equations is derived. Detailed consideration is given to the case of plane deformation. A two-dimensional system of equations of plane deformation is written in the complex form and its general solution is represented by means of three analytic functions of a complex variable and two solutions of Helmholtz equations. The constructed general solution enables one to solve analytically a sufficiently wide class of plane boundary value problems of the elastic equilibrium of porous Cosserat media with double porosity. A concrete boundary value problem for a concentric ring is solved.
\end{abstract}

\section{Introduction}

A model of elastic equilibrium of porous media with double porosity was constructed in the works [1-3]. The theory justified in these papers combines the previously proposed model of Barenblatt for media with double porosity [4] and that of Biot for media with ordinary porosity [5]. For a detailed account of the development of the theory of porous media and relevant references, see [6]. Various issues related to the elastic equilibrium of bodies with double porosities are treated in [7-15].

It should be noted that all the papers mentioned above dealt with a classical (symmetric) medium. But we do not know of any works where problems of double porous elasticity would have been considered for a nonsymmetric elastic Cosserat medium [16-34]. In our opinion, the investigation of such problems is interesting from both theoretical and practical standpoints. For this reason, we considered the elastic equilibrium of porous bodies with double porosity in the case of the nonsymmetric Cosserat theory.

In Section 2 we give the basic three-dimensional equations of the static equilibrium of porous elastic materials with double porosity in the case of an elastic Cosserat medium. In Section 3 we consider the case of a plane deformed state and write the corresponding two-dimensional system of equilibrium equations in the complex form. In Section 4 we construct the general solution of the abovementioned system of equations by means of analytic functions of a complex variable and solutions of Helmholtz equations. The obtained analogues of the Kolosov-Muskhelishvili formulas [35] make it possible to solve analytically plane boundary value problems of the elastic equilibrium of porous Cosserat media with double porosity. Finally, in Section 5 we solve a boundary value problem for a concentric circular ring.

\section{Basic Three-Dimensional Relations}

Let an elastic body with double porosity occupy the domain $\bar{\Omega} \subset R^{3}$. Denote by $\left(x_{1}, x_{2}, x_{3}\right)$ a point of the domain $\bar{\Omega}$ in the Cartesian coordinate system. Let the domain $\bar{\Omega}$ be filled with an elastic Cosserat medium having double porosity. The considered solid body is characterized by the displacement vector $\mathbf{u}=\left(u_{1}, u_{2}, u_{3}\right)$, rotation vector $\boldsymbol{\omega}=\left(\omega_{1}, \omega_{2}, \omega_{3}\right)$, and also the fluid pressures $p_{1}\left(x_{1}, x_{2}, x_{3}\right)$ and $p_{2}\left(x_{1}, x_{2}, x_{3}\right)$ occurring, respectively, in the pores and fissures of the porous medium. 
Then a homogeneous system of static equilibrium equations is written in the form [25]

$$
\begin{gathered}
\partial_{i} \sigma_{i j}=0, \\
\partial_{i} \mu_{i j}+\epsilon_{j i k} \sigma_{i k}=0, \quad j=1,2,3
\end{gathered}
$$

in $\Omega$,

where $\sigma_{i j}$ are stress tensor components, $\mu_{i j}$ are moment stress tensor components, $\epsilon_{i j k}$ is the Levi-Civita symbol, and $\partial_{i} \equiv$ $\partial / \partial x_{i}$; the summation over the recurring index $i$ is assumed to be done from 1 to 3 .

Formulas that interrelate the stress and moment stress components, the displacement and rotation vector components, and the pressures $p_{1}, p_{2}$ have the form $[13,25]$

$$
\begin{aligned}
& \sigma_{i j}=\left(\lambda \operatorname{div} \mathbf{u}-\beta_{1} p_{1}-\beta_{2} p_{2}\right) \delta_{i j}+(\mu+\alpha) \partial_{i} u_{j} \\
&+(\mu-\alpha) \partial_{j} u_{i}-2 \alpha \epsilon_{i j k} \omega_{k}, \\
& \mu_{i j}= \sigma \operatorname{div} \boldsymbol{\omega} \delta_{i j}+(\nu+\beta) \partial_{i} \omega_{j}+(\nu-\beta) \partial_{j} \omega_{i}, \\
& \quad j=1,2,3,
\end{aligned}
$$

where $\lambda, \mu$ are the Lamé parameters, $\alpha, \beta, \nu, \sigma$ are the constants characterizing the microstructure of the considered elastic medium, $\beta_{1}$ and $\beta_{2}$ are the effective stress parameters, and $\delta_{i j}$ is the Kronecker delta.

As is well known, for the internal energy to be positive it is necessary that the following conditions be fulfilled [28]:

$$
\begin{aligned}
3 \lambda+2 \mu+\alpha & \geq 0, \\
2 \mu+\alpha & \geq 0, \\
\alpha & \geq 0, \\
3 \sigma+2 \nu & \geq 0, \\
|\nu-\beta| & \leq \nu+\beta .
\end{aligned}
$$

In the stationary case, the values $p_{1}$ and $p_{2}$ satisfy the following system of [13]

$$
\begin{aligned}
& \left(k_{1} \Delta-\gamma\right) p_{1}+\left(k_{12} \Delta+\gamma\right) p_{2}=0 \\
& \left(k_{21} \Delta+\gamma\right) p_{1}+\left(k_{2} \Delta-\gamma\right) p_{2}=0
\end{aligned}
$$

in $\Omega$,

where $k_{1}=\kappa_{1} / \mu^{\prime}, k_{2}=\kappa_{2} / \mu^{\prime}, k_{12}=\kappa_{12} / \mu^{\prime}$, and $k_{21}=\kappa_{21} / \mu^{\prime}$, $\mu^{\prime}$ is fluid viscosity, $\kappa_{1}$ and $\kappa_{2}$ are the macroscopic intrinsic permeabilities associated with matrix and fissure porosity, $\kappa_{12}$ and $\kappa_{21}$ are the cross-coupling permeabilities for fluid flow at the interface between the matrix and fissure phases, $\gamma>0$ is the internal transport coefficient and corresponds to fluid transfer rate with respect to the intensity of flow between the pore and fissures, and $\Delta \equiv \partial_{11}+\partial_{22}+\partial_{33}$ is the threedimensional Laplace operator.

The three-dimensional system of (1), (2), and (4) describes the static equilibrium of a porous elastic Cosserat medium with double porosity. Substituting relations (2) into (1), we obtain equilibrium equations with respect to the components of the displacement and rotation vectors:

$$
\begin{aligned}
& (\mu+\alpha) \Delta u_{j}+(\lambda+\mu-\alpha) \partial_{j}\left(\partial_{k} u_{k}\right)-2 \alpha \epsilon_{i j k} \partial_{i} \omega_{k} \\
& \quad-\partial_{j}\left(\beta_{1} p_{1}+\beta_{2} p_{2}\right)=0, \\
& (\nu+\beta) \Delta \omega_{j}+(\sigma+\nu-\beta) \partial_{j}\left(\partial_{k} \omega_{k}\right)+2 \alpha \epsilon_{j i k} \partial_{i} u_{k} \\
& \quad-4 \alpha \omega_{j}=0, \quad j=1,2,3
\end{aligned}
$$

in $\Omega$.

If we add boundary conditions on the boundary $\partial \Omega$ of the domain $\Omega$, to the system of equilibrium equations, then we can consider various classical boundary value problems.

The following lemma is easy to prove.

Lemma 1. If $\gamma>0, k_{1} k_{2}-k_{12} k_{21}>0$, then the system of (4) is equivalent to two independent equations: to the Laplace equation

$$
\Delta\left[\left(k_{1}+k_{21}\right) p_{1}+\left(k_{2}+k_{12}\right) p_{2}\right]=0
$$

with respect to the combination $\left(k_{1}+k_{21}\right) p_{1}+\left(k_{2}+k_{12}\right) p_{2}$ and to the Helmholtz equation with respect to the difference $p_{1}-p_{2}$

$$
\Delta\left(p_{1}-p_{2}\right)-\varsigma^{2}\left(p_{1}-p_{2}\right)=0,
$$

where $^{2}:=\gamma\left(k_{1}+k_{2}+k_{12}+k_{21}\right) /\left(k_{1} k_{2}-k_{12} k_{21}\right)>0$.

Proof. Adding the first equation of system (4) to the second equation of this system, we immediately obtain (6).

Let us write system (4) in the matrix form:

$$
\left(\begin{array}{ll}
k_{1} & k_{12} \\
k_{21} & k_{2}
\end{array}\right)\left(\begin{array}{c}
\Delta p_{1} \\
\Delta p_{2}
\end{array}\right)-\gamma\left(\begin{array}{cc}
1 & -1 \\
-1 & 1
\end{array}\right)\left(\begin{array}{l}
p_{1} \\
p_{2}
\end{array}\right)=\left(\begin{array}{l}
0 \\
0
\end{array}\right) .
$$

By assumption, the determinant of the matrix $\operatorname{det}\left(\begin{array}{cc}k_{1} & k_{12} \\ k_{21} & k_{2}\end{array}\right)=$ $k_{1} k_{2}-k_{12} k_{21}>0$ is positive. The left multiplication of all members of the latter equation by the matrix $\left(\begin{array}{ll}k_{1} & k_{12} \\ k_{21} & k_{2}\end{array}\right)^{-1}$ gives the system

$$
\begin{aligned}
& \Delta p_{1}-\frac{\gamma\left(k_{2}+k_{12}\right)}{k_{1} k_{2}-k_{12} k_{21}}\left(p_{1}-p_{2}\right)=0 \\
& \Delta p_{2}+\frac{\gamma\left(k_{1}+k_{21}\right)}{k_{1} k_{2}-k_{12} k_{21}}\left(p_{1}-p_{2}\right)=0 .
\end{aligned}
$$

If from the first equation of the latter system we subtract the second equation of this system, then we obtain (7).

Since the transformation determinant is defined as

$$
\begin{aligned}
& \operatorname{det}\left(\begin{array}{cc}
k_{1}+k_{21} & k_{2}+k_{12} \\
1 & -1
\end{array}\right)=-\left(k_{1}+k_{2}+k_{12}+k_{21}\right) \\
& \quad<0
\end{aligned}
$$

the validity of the lemma is proved. 
Corollary 2. If on the boundary $\partial \Omega$ of the domain $\Omega, p_{1}=p_{2}$, then $p_{1}=p_{2}$ throughout the body $\bar{\Omega}$.

This corollary follows from the fact that the homogeneous Helmholtz equation (7) with zero boundary conditions has only the trivial solution.

\section{The Plane Deformation Case}

From the basic three-dimensional equations we obtain the basic equations for the case of plane deformation. Let $\Omega$ be a sufficiently long cylindrical body with generatrix parallel to the $x_{3}$-axis. Denote by $V$ the cross section of this cylindrical body; thus $V \subset R^{2}$. In the case of plane deformation $u_{3}=0$, $\omega_{1}=0$, and $\omega_{2}=0$, while the functions $u_{1}, u_{2}, \omega_{3}, p_{1}$, and $p_{2}$ do not depend on the coordinate $x_{3}$ (see, e.g., [29]).

As follows from formulas (2), in the case of plane deformation

$$
\begin{aligned}
\sigma_{\alpha 3} & =0, \\
\sigma_{3 \alpha} & =0, \\
\mu_{\alpha \beta} & =0, \\
\mu_{33} & =0, \\
\alpha & =1,2 ; \\
\beta & =1,2 .
\end{aligned}
$$

Therefore the system of equilibrium equations (1) takes the form

$$
\begin{aligned}
\partial_{1} \sigma_{11}+\partial_{2} \sigma_{21} & =0 \\
\partial_{1} \sigma_{12}+\partial_{2} \sigma_{22} & =0 \\
\partial_{1} \mu_{13}+\partial_{2} \mu_{23}+\left(\sigma_{12}-\sigma_{21}\right) & =0
\end{aligned}
$$

in $V$.

Relations (2) are rewritten as

$$
\begin{aligned}
& \sigma_{11}=-\beta_{1} p_{1}-\beta_{2} p_{2}+\lambda \theta+2 \mu \partial_{1} u_{1}, \\
& \sigma_{22}=-\beta_{1} p_{1}-\beta_{2} p_{2}+\lambda \theta+2 \mu \partial_{2} u_{2}, \\
& \sigma_{12}=(\mu+\alpha) \partial_{1} u_{2}+(\mu-\alpha) \partial_{2} u_{1}-2 \alpha \omega_{3}, \\
& \sigma_{21}=(\mu+\alpha) \partial_{2} u_{1}+(\mu-\alpha) \partial_{1} u_{2}+2 \alpha \omega_{3}, \\
& \sigma_{33}=-\beta_{1} p_{1}-\beta_{2} p_{2}+\lambda \theta, \\
& \mu_{13}=(v+\beta) \partial_{1} \omega_{3}, \\
& \mu_{23}=(v+\beta) \partial_{2} \omega_{3}, \\
& \mu_{31}=(v-\beta) \partial_{1} \omega_{3}, \\
& \mu_{32}=(v-\beta) \partial_{2} \omega_{3},
\end{aligned}
$$

where $\theta:=\partial_{1} u_{1}+\partial_{2} u_{2}$.
Equations (6) and (7) take the form

$$
\begin{aligned}
& \Delta_{2}\left[\left(k_{1}+k_{21}\right) p_{1}+\left(k_{2}+k_{12}\right) p_{2}\right]=0 \text { in } V, \\
& \Delta_{2}\left(p_{1}-p_{2}\right)-\varsigma^{2}\left(p_{1}-p_{2}\right)=0 \text { in } V \text {, }
\end{aligned}
$$

where $\Delta_{2}:=\partial_{11}+\partial_{22}$ is the Laplace operator in two dimensions.

If relations (13) are substituted into system (12), then we obtain the following system of equilibrium equations with respect to the functions $u_{1}, u_{2}$, and $\omega_{3}$ :

$$
\begin{aligned}
& (\mu+\alpha) \Delta_{2} u_{1}+(\lambda+\mu-\alpha) \partial_{1} \theta+2 \alpha \partial_{2} \omega_{3} \\
& \quad-\partial_{1}\left(\beta_{1} p_{1}+\beta_{2} p_{2}\right)=0, \\
& (\mu+\alpha) \Delta_{2} u_{2}+(\lambda+\mu-\alpha) \partial_{2} \theta-2 \alpha \partial_{1} \omega_{3} \\
& \quad-\partial_{2}\left(\beta_{1} p_{1}+\beta_{2} p_{2}\right)=0, \\
& (\nu+\beta) \Delta_{2} \omega_{3}+2 \alpha\left(\partial_{1} u_{2}-\partial_{2} u_{1}\right)-4 \alpha \omega_{3}=0
\end{aligned}
$$

in $V$.

On the plane $x_{1} x_{2}$, we introduce the complex variable $z=$ $x_{1}+i x_{2}=r e^{i \alpha}\left(i^{2}=-1\right)$ and the operators $\partial_{z}=0.5\left(\partial_{1}-i \partial_{2}\right)$, $\partial_{\bar{z}}=0.5\left(\partial_{1}+i \partial_{2}\right), \bar{z}=x_{1}-i x_{2}$, and $\Delta_{2}=4 \partial_{z} \partial_{\bar{z}}$.

To write system (12) in the complex form, the second equation of this system is multiplied by $i$ and summed up with the first equation [35-37] (see also [38, 39]):

$$
\begin{aligned}
& \partial_{z}\left(\sigma_{11}-\sigma_{22}+i\left(\sigma_{12}+\sigma_{21}\right)\right) \\
& \quad+\partial_{\bar{z}}\left(\sigma_{11}+\sigma_{22}+i\left(\sigma_{12}-\sigma_{21}\right)\right)=0, \\
& \partial_{z}\left(\mu_{13}+i \mu_{23}\right)+\partial_{\bar{z}}\left(\mu_{13}-i \mu_{23}\right)+\sigma_{12}-\sigma_{21}=0
\end{aligned}
$$

in $V$,

where, by formulas (13),

$$
\begin{aligned}
\sigma_{11}- & \sigma_{22}+i\left(\sigma_{12}+\sigma_{21}\right)=4 \mu \partial_{\bar{z}} u_{+}, \\
\sigma_{11} & \sigma_{22}+i\left(\sigma_{12}-\sigma_{21}\right) \\
= & 2(\lambda+\mu-\alpha) \theta+4 \alpha \partial_{z} u_{+}-4 \alpha i \omega_{3} \\
& -2\left(\beta_{1} p_{1}+\beta_{2} p_{2}\right), \\
& +i \mu_{23}=2(\nu+\beta) \partial_{\bar{z}} \omega_{3}, \\
\mu_{31} & +i \mu_{32}=2(\nu-\beta) \partial_{\bar{z}} \omega_{3} ; \\
u_{+}:= & u_{1}+i u_{2} ; \\
\theta= & \partial_{z} u_{+}+\partial_{\bar{z}} \bar{u}_{+} .
\end{aligned}
$$

We write (14) and (15) as

$$
\begin{aligned}
& \partial_{\bar{z}} \partial_{z}\left[\left(k_{1}+k_{21}\right) p_{1}+\left(k_{2}+k_{12}\right) p_{2}\right]=0 \text { in } V \text {, } \\
& 4 \partial_{\bar{z}} \partial_{z}\left(p_{1}-p_{2}\right)-\varsigma^{2}\left(p_{1}-p_{2}\right)=0 \quad \text { in } V \text {. }
\end{aligned}
$$


If relations (18) are substituted into system (17), then system (16) is written in the complex form:

$$
\begin{aligned}
& 2(\mu+\alpha) \partial_{\bar{z}} \partial_{z} u_{+}+(\lambda+\mu-\alpha) \partial_{\bar{z}} \theta-2 \alpha i \partial_{\bar{z}} \omega_{3} \\
& \quad-\partial_{\bar{z}}\left(\beta_{1} p_{1}+\beta_{2} p_{2}\right)=0 \\
& 2(\nu+\beta) \partial_{\bar{z}} \partial_{z} \omega_{3}+\alpha i\left(\theta-2 \partial_{z} u_{+}\right)-2 \alpha \omega_{3}=0
\end{aligned}
$$

in $V$.

\section{The General Solution of System (19)-(20)}

In this section, we construct the analogues of the KolosovMuskhelishvili formulas [35] (see also [36-39]) for system (19)-(20).

Equations (19) imply that

$$
\begin{aligned}
\left(k_{1}+k_{21}\right) p_{1}+\left(k_{2}+k_{12}\right) p_{2} & =k_{0}\left[f^{\prime}(z)+\overline{f^{\prime}(z)}\right], \\
p_{1}-p_{2} & =k_{0} \eta(z, \bar{z}),
\end{aligned}
$$

where $k_{0}:=k_{1}+k_{2}+k_{12}+k_{21}, f(z)$ is an arbitrary analytic function of a complex variable $z$ in the domain $V$, and $\eta(z, \bar{z})$ is an arbitrary solution of the Helmholtz equation

$$
4 \partial_{z} \partial_{\bar{z}} \eta-\varsigma^{2} \eta=0
$$

From system (21) we easily obtain the expressions for the pressures $p_{1}$ and $p_{2}$ :

$$
\begin{aligned}
& p_{1}=f^{\prime}(z)+\overline{f^{\prime}(z)}+\left(k_{2}+k_{12}\right) \eta(z, \bar{z}), \\
& p_{2}=f^{\prime}(z)+\overline{f^{\prime}(z)}-\left(k_{1}+k_{21}\right) \eta(z, \bar{z}) .
\end{aligned}
$$

Theorem 3. The general solution of the system of (20) is represented as follows:

$$
\begin{aligned}
2 \mu u_{+}= & \kappa \varphi(z)-z \overline{\varphi^{\prime}(z)}-\overline{\psi(z)}+2 i \partial_{\bar{z}} \chi(z, \bar{z}) \\
& +\frac{\mu\left(\beta_{1}+\beta_{2}\right)}{\lambda+2 \mu}\left(f(z)+z \overline{f^{\prime}(z)}\right) \\
& +\delta \partial_{\bar{z}} \eta(z, \bar{z}), \\
2 \mu \omega_{3}= & \frac{2 \mu}{v+\beta} \chi(z, \bar{z})-\frac{\kappa+1}{2} i\left(\varphi^{\prime}(z)-\overline{\varphi^{\prime}(z)}\right),
\end{aligned}
$$

where $\kappa=(\lambda+3 \mu) /(\lambda+\mu), \delta:=4 \mu\left(\left(k_{2}+k_{12}\right) \beta_{1}-\right.$ $\left.\left(k_{1}+k_{21}\right) \beta_{2}\right) /(\lambda+2 \mu) \varsigma^{2}, \varphi(z)$ and $\psi(z)$ are arbitrary analytic functions of a complex variable $z$ in the domain $V$, and $\chi(z, \bar{z})$ is an arbitrary solution of the Helmholtz equation

$$
4 \partial_{z} \partial_{\bar{z}} \chi-\xi^{2} \chi=0
$$

where

$$
\xi^{2}:=\frac{4 \mu \alpha}{(\nu+\beta)(\mu+\alpha)}>0
$$

Proof. We take the operator $\partial_{\bar{z}}$ out of the brackets in the lefthand part of the first equation of system (20):

$$
\begin{gathered}
\partial_{\bar{z}}\left(2(\mu+\alpha) \partial_{z} u_{+}+(\lambda+\mu-\alpha) \theta-2 \alpha i \omega_{3}\right. \\
\left.-\left(\beta_{1} p_{1}+\beta_{1} p_{1}\right)\right)=0 .
\end{gathered}
$$

Since (28) is a system of Cauchy-Riemann equations, we have

$$
\begin{gathered}
2(\mu+\alpha) \partial_{z} u_{+}+(\lambda+\mu-\alpha) \theta-2 \alpha i \omega_{3} \\
=(\kappa+1) \varphi^{\prime}(z)+\beta_{1} p_{1}+\beta_{2} p_{2}
\end{gathered}
$$

where $\varphi(z)$ is an arbitrary analytic function of $z$.

A conjugate equation to (29) has the form

$$
\begin{gathered}
2(\mu+\alpha) \partial_{\bar{z}} \bar{u}_{+}+(\lambda+\mu-\alpha) \theta+2 \alpha i \omega_{3} \\
=(\kappa+1) \overline{\varphi^{\prime}(z)}+\beta_{1} p_{1}+\beta_{2} p_{2} .
\end{gathered}
$$

Summing up (29) and (30) and taking into account that

$$
\theta=\partial_{z} u_{+}+\partial_{\bar{z}} \bar{u}_{+}
$$

we obtain

$$
\theta=\frac{1}{\lambda+\mu}\left(\varphi^{\prime}(z)+\overline{\varphi^{\prime}(z)}\right)+\frac{1}{\lambda+2 \mu}\left(\beta_{1} p_{1}+\beta_{2} p_{2}\right) .
$$

If from (29) we subtract (30) and write the expression $i\left(\partial_{z} u_{+}-\right.$ $\left.\partial_{\bar{z}} \bar{u}_{+}\right)$, then we have

$$
\begin{aligned}
i\left(\partial_{z} u_{+}-\partial_{\bar{z}} \bar{u}_{+}\right)= & \frac{\kappa+1}{2(\mu+\alpha)} i\left(\varphi^{\prime}(z)-\overline{\varphi^{\prime}(z)}\right) \\
& -\frac{2 \alpha}{\mu+\alpha} \omega_{3} .
\end{aligned}
$$

The second equation of system (20) is written as

$$
4 \partial_{z} \partial_{\bar{z}} \omega_{3}-\frac{2 \alpha}{\nu+\beta} i\left(\partial_{z} u_{+}-\partial_{\bar{z}} \bar{u}_{+}\right)-\frac{4 \alpha}{\nu+\beta} \omega_{3}=0 .
$$

Substituting formula (33) into formula (34) we obtain the equation

$$
\Delta_{2} \omega_{3}-\xi^{2} \omega_{3}=\frac{\alpha(\kappa+1)}{(\nu+\beta)(\mu+\alpha)} i\left(\varphi^{\prime}(z)-\overline{\varphi^{\prime}(z)}\right) .
$$

The general solution of (35) is written in the form

$$
2 \mu \omega_{3}=\frac{2 \mu}{v+\beta} \chi(z, \bar{z})-\frac{\kappa+1}{2} i\left(\varphi^{\prime}(z)-\overline{\varphi^{\prime}(z)}\right),
$$

where $\chi(z, \bar{z})$ is a general solution of the Helmholtz equation

$$
\Delta_{2} \chi-\xi^{2} \chi=0
$$

The multiplier $2 \mu /(\nu+\beta)$ has been introduced for convenience in writing our subsequent formulas. 
Substituting formulas (32) and (36) into (29) and taking into account that $\chi(z, \bar{z})$ is a solution of $(37)$, we obtain

$$
\begin{aligned}
2 \mu \partial_{z} u_{+}= & \kappa \varphi^{\prime}(z)-\overline{\varphi^{\prime}(z)}+2 i \partial_{z} \partial_{\bar{z}} \chi(z, \bar{z}) \\
& +\frac{\mu}{\lambda+2 \mu}\left(\beta_{1} p_{1}+\beta_{2} p_{2}\right) .
\end{aligned}
$$

From formulas (23) we find the following expression for the combination $\beta_{1} p_{1}+\beta_{2} p_{2}$ :

$$
\begin{aligned}
& \frac{\mu}{\lambda+2 \mu}\left(\beta_{1} p_{1}+\beta_{2} p_{2}\right) \\
& \quad=\frac{\mu\left(\beta_{1}+\beta_{2}\right)}{\lambda+2 \mu}\left(f^{\prime}(z)+\overline{f^{\prime}(z)}\right)+\delta \partial_{z} \partial_{\bar{z}} \eta(z, \bar{z}) .
\end{aligned}
$$

Substituting the latter formula into (38), integrating over $z$, and using

$$
\eta=\frac{4}{\varsigma^{2}} \partial_{\bar{z}} \partial_{z} \eta
$$

we obtain formula (24) which we are proving:

$$
\begin{aligned}
2 \mu u_{+}= & \kappa \varphi(z)-z \overline{\varphi^{\prime}(z)}-\overline{\psi(z)}+2 i \partial_{\bar{z}} \chi(z, \bar{z}) \\
& +\frac{\mu\left(\beta_{1}+\beta_{2}\right)}{\lambda+2 \mu}\left(f(z)+z \overline{f^{\prime}(z)}\right) \\
& +\delta \partial_{\bar{z}} \eta(z, \bar{z}) .
\end{aligned}
$$

Thus, if the solution of system (20) is sufficiently smooth, then it is represented in the form of (24) and (25). Conversely, if expressions (24) and (25) are substituted into (20), then this system will be satisfied.

Substituting expressions (24) and (25) into formulas (18), for combinations of stress tensor components we obtain the following formulas:

$$
\begin{aligned}
& \sigma_{11}+\sigma_{22}+i\left(\sigma_{12}-\sigma_{21}\right)=2\left[\varphi^{\prime}(z)+\overline{\varphi^{\prime}(z)}\right. \\
& -2 i \partial_{z} \partial_{\bar{z}} \chi-\frac{\mu\left(\beta_{1}+\beta_{2}\right)}{\lambda+2 \mu}\left(f^{\prime}(z)+\overline{f^{\prime}(z)}\right) \\
& \left.-\delta \partial_{z} \partial_{\bar{z}} \eta\right], \\
& \sigma_{11}-\sigma_{22}+i\left(\sigma_{12}+\sigma_{21}\right)=2\left[-z \overline{\varphi^{\prime \prime}(z)}-\overline{\psi^{\prime}(z)}\right. \\
& \left.+2 i \partial_{\bar{z}} \partial_{\bar{z}} \chi\right]+\frac{2 \mu\left(\beta_{1}+\beta_{2}\right)}{\lambda+2 \mu} \overline{f^{\prime \prime}(z)}+2 \delta \partial_{\bar{z}} \partial_{\bar{z}} \eta, \\
& \sigma_{33}=\frac{\lambda}{\lambda+\mu}\left[\varphi^{\prime}(z)+\overline{\varphi^{\prime}(z)}\right]
\end{aligned}
$$

$$
\begin{aligned}
& -\frac{2 \mu}{\lambda+2 \mu}\left[\left(\beta_{1}+\beta_{2}\right)\left(f^{\prime}(z)+\overline{f^{\prime}(z)}\right)+\delta \chi(z, \bar{z})\right] \\
& \mu_{13}+i \mu_{23}=2 \partial_{\bar{z}} \chi+\frac{(\kappa+1)(\nu+\beta)}{2 \mu} \overline{i \varphi^{\prime \prime}(z)} \\
& \mu_{31}+i \mu_{32}=\frac{2(\nu-\beta)}{\nu+\beta} \partial_{\bar{z}} \chi+\frac{(\kappa+1)(\nu-\beta)}{2 \mu} i \overline{\varphi^{\prime \prime}(z)} .
\end{aligned}
$$

Thus, the general solution of a two-dimensional system of differential equations that describes the static equilibrium of a porous elastic medium with double porosity is represented by means of three analytic functions of a complex variable and two solutions of the Helmholtz equation. By an appropriate choice of these functions we can satisfy five independent classical boundary conditions.

Let mutually perpendicular unit vectors $\mathbf{l}$ and $\mathbf{s}$ be such that

$$
\mathbf{l} \times \mathbf{s}=\mathbf{e}_{3}
$$

where $\mathbf{e}_{3}$ is the unit vector directed along the $x_{3}$-axis. The vector 1 forms the angle $\vartheta$ with the positive direction of the $x_{1}$ axis. Then the displacement components $u_{l}=\mathbf{u} \cdot \mathbf{l}, u_{s}=\mathbf{u} \cdot \mathbf{s}$, as well as the stress and moment stress components acting on an area of arbitrary orientation, are expressed by the formulas

$$
\begin{aligned}
& u_{l}+i u_{s}=e^{-i \vartheta} u_{+} \text {, } \\
& \sigma_{l l}+i \sigma_{l s}=0.5\left[\sigma_{11}+\sigma_{22}+i\left(\sigma_{12}-\sigma_{21}\right)\right. \\
& \left.+\left(\sigma_{11}-\sigma_{22}+i\left(\sigma_{12}+\sigma_{21}\right)\right) e^{-2 i \vartheta}\right] \text {, } \\
& \mu_{l 3}=0.5\left[\left(\mu_{13}+i \mu_{23}\right) e^{-i \vartheta}+\left(\mu_{13}-i \mu_{23}\right) e^{i \vartheta}\right] \text {. }
\end{aligned}
$$

\section{A Problem for a Concentric Circular Ring}

In this section, we solve a concrete boundary value problem for a concentric circular ring. On the boundary of the considered domain which is free from stresses and moment stresses, the values of pressures $p_{1}$ and $p_{2}$ are given.

Let a porous elastic body with double porosity occupy the domain $V$ which is bounded by the concentric circumferences $L_{1}$ and $L_{2}$ with radii $R_{1}$ and $R_{2}$, respectively $\left(R_{1}<R_{2}\right)$ (Figure 1).

We consider the following problem:

$$
\begin{array}{r}
\sigma_{r r}-i \sigma_{r \alpha}= \begin{cases}0, & r=R_{1}, \\
0, & r=R_{2},\end{cases} \\
\mu_{r 3}= \begin{cases}0, & r=R_{1}, \\
0, & r=R_{2},\end{cases} \\
\left(k_{1}+k_{21}\right) p_{1}+\left(k_{2}+k_{12}\right) p_{2}= \begin{cases}p^{(1)}, & r=R_{1}, \\
p^{(2)}, & r=R_{2},\end{cases}
\end{array}
$$




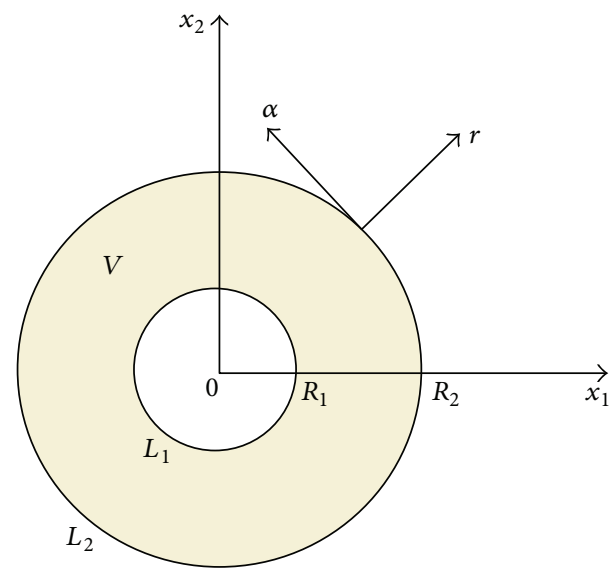

FIGURE 1: The considered circular ring.

$$
p_{1}-p_{2}= \begin{cases}0, & r=R_{1}, \\ 2 p \cos \alpha, & r=R_{2}\end{cases}
$$

where $p^{(1)}, p^{(2)}$, and $p$ are given constant values.

Since $k_{1} p_{1}+k_{2} p_{2}$ satisfy the Laplace equation (see formula (14)), having satisfied the boundary conditions (46), we obtain

$$
\begin{aligned}
& \left(k_{1}+k_{21}\right) p_{1}+\left(k_{2}+k_{12}\right) p_{2} \\
& =\frac{p^{(2)}-p^{(1)}}{\ln R_{2}-\ln R_{1}} \ln r+\frac{p^{(1)} \ln R_{2}-p^{(2)} \ln R_{1}}{\ln R_{2}-\ln R_{1}} .
\end{aligned}
$$

The analytic function $f^{\prime}(z)$ is written in the form

$$
\begin{gathered}
f^{\prime}(z)=\frac{1}{2 k_{0}}\left(\frac{p^{(2)}-p^{(1)}}{\ln R_{2}-\ln R_{1}} \ln z\right. \\
\left.+\frac{p^{(1)} \ln R_{2}-p^{(2)} \ln R_{1}}{\ln R_{2}-\ln R_{1}}\right) .
\end{gathered}
$$

The metaharmonic function $\eta(z, \bar{z})$ is represented as a series:

$$
\eta(z, \bar{z})=\frac{1}{k_{0}} \sum_{-\infty}^{+\infty}\left(\alpha_{n} I_{n}(\varsigma r)+\beta_{n} K_{n}(\varsigma r)\right) e^{i n \alpha}
$$

where $I_{n}(\varsigma r)$ and $K_{n}(\varsigma r)$ are modified Bessel functions of $n$th order.

Using the boundary conditions (47), we obtain

$$
\alpha_{n}=\beta_{n}=0 \quad \text { when } n \neq \pm 1 \text {. }
$$

For the coefficients $\alpha_{1}=\alpha_{-1}$ and $\beta_{1}=\beta_{-1}$ we obtain the following system of equations:

$$
\begin{aligned}
& \alpha_{1} I_{1}\left(\varsigma R_{1}\right)+\beta_{1} K_{1}\left(\varsigma R_{1}\right)=0, \\
& \alpha_{1} I_{1}\left(\varsigma R_{2}\right)+\beta_{1} K_{1}\left(\varsigma R_{2}\right)=p,
\end{aligned}
$$

whence

$$
\begin{aligned}
& \alpha_{1}=-\frac{p K_{1}\left(\varsigma R_{1}\right)}{I_{1}\left(\varsigma R_{1}\right) K_{1}\left(\varsigma R_{2}\right)-I_{1}\left(\varsigma R_{2}\right) K_{1}\left(\varsigma R_{1}\right)}, \\
& \beta_{1}=\frac{p I_{1}\left(\varsigma R_{1}\right)}{I_{1}\left(\varsigma R_{1}\right) K_{1}\left(\varsigma R_{2}\right)-I_{1}\left(\varsigma R_{2}\right) K_{1}\left(\varsigma R_{1}\right)} .
\end{aligned}
$$

Thus

$$
\eta(z, \bar{z})=\frac{2}{k_{0}}\left(\alpha_{1} I_{1}(\varsigma r)+\beta_{1} K_{1}(\varsigma r)\right) \cos \alpha,
$$

where the coefficients $\alpha_{1}$ and $\beta_{1}$ are calculated by formulas (53).

Let us now satisfy the boundary conditions (45). Due to the general representations (42) and (44) obtained above we have

$$
\begin{aligned}
\sigma_{r r}- & i \sigma_{r \alpha} \\
= & \varphi^{\prime}(z)+\overline{\varphi^{\prime}(z)}-e^{2 i \alpha}\left(\bar{z} \varphi^{\prime \prime}(z)+\psi^{\prime}(z)\right) \\
& -\frac{\xi^{2} i}{2}\left(\chi(z, \bar{z})-\frac{4}{\xi^{2}} \partial_{z} \partial_{z} \chi(z, \bar{z}) e^{2 i \alpha}\right) \\
& -\frac{\delta \varsigma^{2}}{4}\left(\eta(z, \bar{z})-\frac{4}{\varsigma^{2}} \partial_{z} \partial_{z} \eta(z, \bar{z}) e^{2 i \alpha}\right) \\
& -\frac{\mu\left(\beta_{1}+\beta_{2}\right)}{\lambda+2 \mu}\left(f^{\prime}(z)+\overline{f^{\prime}(z)}-\bar{z} f^{\prime \prime}(z) e^{2 i \alpha}\right) \\
= & \left\{\begin{array}{l}
0, \quad r=R_{1}, \\
0, \quad r=R_{2} ;
\end{array}\right.
\end{aligned}
$$

$\mu_{r 3}$

$$
\begin{aligned}
= & \frac{(\kappa+1)(\nu+\beta) i}{4 \mu}\left(\overline{\varphi^{\prime \prime}(z)} e^{-i \alpha}-\varphi^{\prime \prime}(z) e^{i \alpha}\right) \\
& +\partial_{\bar{z}} \chi(z, \bar{z}) e^{-i \alpha}+\partial_{z} \chi(z, \bar{z}) e^{i \alpha} \\
= & \begin{cases}0, & r=R_{1}, \\
0, & r=R_{2} .\end{cases}
\end{aligned}
$$

The analytic functions $\varphi^{\prime}(z)$ and $\psi^{\prime}(z)$ are represented as series:

$$
\begin{aligned}
& \varphi^{\prime}(z)=A \ln z+\sum_{-\infty}^{+\infty} a_{n} r^{n} e^{i n \alpha}, \\
& \psi^{\prime}(z)=\sum_{-\infty}^{+\infty} b_{n} r^{n} e^{i n \alpha} .
\end{aligned}
$$

From the first condition of displacement uniqueness it follows that $A=0$. It is also assumed that $a_{0}$ is a real value; that is, $a_{0}=\bar{a}_{0}$ (see [35]).

The metaharmonic function $\chi(z, \bar{z})$ is sought as a series

$$
\chi(z, \bar{z})=\sum_{-\infty}^{+\infty}\left(\gamma_{n} I_{n}(\xi r)+\delta_{n} K_{n}(\xi r)\right) e^{i n \alpha}
$$


If representations (56), (57), (54), and (49) are substituted into the boundary conditions (55), then we obtain

$$
\begin{aligned}
& {\left[\sum_{-\infty}^{+\infty}(1-n) a_{n} r^{n} e^{i n \alpha}+\sum_{-\infty}^{+\infty} \bar{a}_{n} r^{n} e^{-i n \alpha}-\sum_{-\infty}^{+\infty} b_{n-2} r^{n-2} e^{i n \alpha}\right.} \\
& -\frac{\xi i}{r} \\
& \left.\cdot \sum_{-\infty}^{+\infty}(1-n)\left(\gamma_{n} I_{n-1}(\xi r)-\delta_{n} K_{n-1}(\xi r)\right) e^{i n \alpha}\right]_{r=R_{\gamma}} \\
& =A_{\gamma}, \quad \gamma=1,2, \\
& {\left[\frac { ( \kappa + 1 ) ( \nu - \beta ) i } { 4 \mu } \left(\sum_{-\infty}^{+\infty} n a_{n} r^{n-1} e^{i n \alpha}\right.\right.} \\
& \left.+\sum_{-\infty}^{+\infty} n \bar{a}_{n} r^{n-1} e^{-i n \alpha}\right) \\
& +\frac{\xi}{2}\left(\sum_{-\infty}^{+\infty} \gamma_{n}\left(I_{n+1}(\xi r)+I_{n-1}(\xi r)\right) e^{i n \alpha}\right. \\
& \left.\left.-\sum_{-\infty}^{+\infty} \delta_{n}\left(K_{n+1}(\xi r)+K_{n-1}(\xi r)\right) e^{i n \alpha}\right)\right]_{r=R_{\gamma}}=0, \\
& \gamma=1,2,
\end{aligned}
$$

where we have introduced the notation

$$
\begin{aligned}
& A_{\gamma}:=\frac{\delta \varsigma}{k_{0} R_{\gamma}}\left[\alpha_{1} I_{2}\left(\varsigma R_{\gamma}\right)-\beta_{1} K_{2}\left(\varsigma R_{\gamma}\right)\right] e^{-i \alpha} \\
&+\frac{\mu\left(\beta_{1}+\beta_{2}\right)}{2(\lambda+2 \mu) k_{0}} \\
& \cdot \frac{\left(p^{(2)}-p^{(1)}\right)\left(2 \ln R_{\gamma}-1\right)+2 p^{(1)} \ln R_{2}-2 p^{(2)} \ln R_{1}}{\ln R_{2}-\ln R_{1}}, \\
& \quad \gamma=1,2 .
\end{aligned}
$$

Equating the free members on both sides of equalities (58) and (59), we obtain the system

$$
\begin{aligned}
2 a_{0}-b_{-2} R_{1}^{-2}-\frac{\xi i}{r}\left(\gamma_{0} I_{1}\left(\xi R_{1}\right)-\delta_{0} K\left(\xi R_{1}\right)\right) & =B_{1} \\
2 a_{0}-b_{-2} R_{2}^{-2}-\frac{\xi i}{r}\left(\gamma_{0} I_{1}\left(\xi R_{2}\right)-\delta_{0} K\left(\xi R_{2}\right)\right) & =B_{2} \\
\gamma_{0} I_{1}\left(\varsigma R_{1}\right)-\delta_{0} K_{1}\left(\varsigma R_{1}\right) & =0 \\
& \gamma_{0} I_{1}\left(\varsigma R_{2}\right)-\delta_{0} K_{1}\left(\varsigma R_{2}\right)=0
\end{aligned}
$$

where

$$
\begin{aligned}
& B_{\gamma}= \frac{\mu\left(\beta_{1}+\beta_{2}\right)}{2(\lambda+2 \mu) k_{0}} \\
& \cdot \frac{\left(p^{(2)}-p^{(1)}\right)\left(2 \ln R_{\gamma}-1\right)+2 p^{(1)} \ln R_{2}-2 p^{(2)} \ln R_{1}}{\ln R_{2}-\ln R_{1}}, \\
& \quad \gamma=1,2 .
\end{aligned}
$$

From system (61) we define the constants $a_{0}, b_{-2}, \gamma_{0}$, and $\delta_{0}$ :

$$
\begin{aligned}
& \gamma_{0}=\delta_{0}=0, \\
& a_{0}=\frac{B_{1} R_{1}^{2}-B_{2} R_{2}^{2}}{2\left(R_{1}^{2}-R_{2}^{2}\right)}, \\
& b_{-2}=\frac{\left(B_{1}-B_{2}\right) R_{1}^{2} R_{2}^{2}}{R_{1}^{2}-R_{2}^{2}} .
\end{aligned}
$$

Equating the coefficients of $e^{i \alpha}$ in (58) gives

$$
\bar{a}_{-1}-b_{-1}=0,
$$

and, using the second displacement uniqueness condition

$$
\kappa a_{-1}+\bar{b}_{-1}=0
$$

we have

$$
\begin{aligned}
& a_{-1}=0, \\
& b_{-1}=0 .
\end{aligned}
$$

Equating the coefficients of $e^{-i \alpha}$ in (58) and (59) and using (66), we obtain the following system of equations:

$$
\begin{aligned}
& \bar{a}_{1} R_{1}-b_{-3} R_{1}^{-3}-\frac{2 \xi i}{R_{1}}\left(\gamma_{-1} I_{2}\left(\xi R_{1}\right)-\delta_{-1} K_{2}\left(\xi R_{1}\right)\right) \\
& =C_{1}, \\
& \bar{a}_{1} R_{2}-b_{-3} R_{2}^{-3}-\frac{2 \xi i}{R_{2}}\left(\gamma_{-1} I_{2}\left(\xi R_{2}\right)-\delta_{-1} K_{2}\left(\xi R_{2}\right)\right) \\
& =C_{2}, \\
& \frac{(\kappa+1)(\nu-\beta)}{4 \mu} \bar{a}_{1}-\xi i\left(\gamma_{-1} I_{1}^{\prime}\left(\xi R_{1}\right)+\delta_{-1} K_{1}^{\prime}\left(\xi R_{1}\right)\right) \\
& \quad=0, \\
& \frac{(\kappa+1)(\nu-\beta)}{4 \mu} \bar{a}_{1}-\xi i\left(\gamma_{-1} I_{1}^{\prime}\left(\xi R_{2}\right)+\delta_{-1} K_{1}^{\prime}\left(\xi R_{2}\right)\right) \\
& \quad=0,
\end{aligned}
$$

where

$$
\begin{aligned}
& C_{\gamma}:=\frac{\delta \varsigma}{k_{0} R_{\gamma}}\left[\alpha_{1} I_{2}\left(\varsigma R_{\gamma}\right)-\beta_{1} K_{2}\left(\varsigma R_{\gamma}\right)\right] ; \\
& I_{1}^{\prime}\left(\xi R_{\gamma}\right)=0.5\left(I_{0}\left(\xi R_{\gamma}\right)+I_{2}\left(\xi R_{\gamma}\right)\right), \\
& K_{1}^{\prime}\left(\xi R_{\gamma}\right)=-0.5\left(K_{0}\left(\xi R_{\gamma}\right)+K_{2}\left(\xi R_{\gamma}\right)\right), \\
& \quad \gamma=1,2 .
\end{aligned}
$$

Excluding $b_{-3}$ from the first two equations of system (67), for defining the coefficients $\bar{a}_{1}, \gamma_{-1}$, and $\delta_{-1}$ we obtain the following system:

$$
\begin{aligned}
& \left(R_{2}^{4}-R_{1}^{4}\right) \bar{a}_{1}-2 \xi i I^{*} \gamma_{-1}+2 \xi i K^{*} \delta_{-1}=C_{2} R_{2}^{3}-C_{1} R_{1}^{3}, \\
& (\kappa+1)(\nu-\beta) \bar{a}_{1}-4 \mu \xi i I_{1}^{\prime}\left(\xi R_{1}\right) \gamma_{-1} \\
& \quad-4 \mu \xi i K_{1}^{\prime}\left(\xi R_{1}\right) \delta_{-1}=0,
\end{aligned}
$$




$$
\begin{aligned}
& (\kappa+1)(\nu-\beta) \bar{a}_{1}-4 \mu \xi i I_{1}^{\prime}\left(\xi R_{2}\right) \gamma_{-1} \\
& -4 \mu \xi i K_{1}^{\prime}\left(\xi R_{2}\right) \delta_{-1}=0
\end{aligned}
$$

where

$$
\begin{aligned}
I^{*} & :=\left(I_{2}\left(\xi R_{2}\right) R_{2}^{2}-I_{2}\left(\xi R_{1}\right) R_{1}^{2}\right), \\
K^{*} & :=\left(K_{2}\left(\xi R_{2}\right) R_{2}^{2}-K_{2}\left(\xi R_{1}\right) R_{1}^{2}\right) .
\end{aligned}
$$

After defining the coefficients $a_{1}, \gamma_{-1}$, and $\delta_{-1}$ from (69), the coefficient $b_{-3}$ is defined from the first or the second equation of system $(67)$. Since $\chi(z, \bar{z})$ is a real function, we have

$$
\begin{aligned}
& \gamma_{1}=\bar{\gamma}_{-1}, \\
& \delta_{1}=\bar{\delta}_{-1} .
\end{aligned}
$$

All other coefficients are equal to zero. Thus the sought functions $\varphi^{\prime}(z), \psi^{\prime}(z)$, and $\chi(z, \bar{z})$ will have the form

$$
\begin{aligned}
\varphi^{\prime}(z) & =a_{0}+a_{1} z, \\
\psi^{\prime}(z) & =\frac{b_{-2}}{z^{2}}+\frac{b_{-3}}{z^{3}}, \\
\chi(z, \bar{z}) & =2 \operatorname{Re}\left[\left(\gamma_{1} I_{1}(\xi r)+\delta_{1} K_{1}(\xi r)\right) e^{i \alpha}\right] .
\end{aligned}
$$

By substituting the obtained values of these functions and functions $f^{\prime}(z)$ and $\eta(z, \bar{z})$ into formulas (42) we find the values of all stress and moment stress components. Though the domain boundary was free from moment stresses, due to fluid pressures in the pores the considered disturbance promoted their appearance inside the domain.

The procedure of solving a boundary value problem remains the same when stresses, moment stresses, and pressures on the domain boundary are given arbitrarily, but the condition that the principal vector and the principal moment of external forces are equal to zero is fulfilled.

\section{Conclusion}

We consider the static equilibrium of porous elastic materials with double porosity for a nonsymmetric elastic Cosserat medium. For the case of plane deformation, a general solution of the corresponding system of differential equations is constructed by means of three analytic functions of a complex variable and two solutions of the Helmholtz equations. The constructed general solution can be applied for solving analytically quite a wide class of boundary value problems. An explicit solution is obtained for a boundary value problem for a concentric circular ring.

In our opinion, problems of double porous elasticity for a nonsymmetric elastic medium may be of interest from theoretical and practical standpoints.

\section{Disclosure}

Any idea in this paper is possessed by the author and may not represent the opinion of Shota Rustaveli National Science Foundation itself.

\section{Competing Interests}

The author declares that there is no conflict of interests.

\section{Acknowledgments}

The designated project has been fulfilled by a financial support of Shota Rustaveli National Science Foundation (Grant SRNSF/FR/358/5-109/14).

\section{References}

[1] R. K. Wilson and E. C. Aifantis, "On the theory of consolidation with double porosity," International Journal of Engineering Science, vol. 20, no. 9, pp. 1009-1035, 1982.

[2] D. E. Beskos and E. C. Aifantis, "On the theory of consolidation with double porosity-II," International Journal of Engineering Science, vol. 24, no. 11, pp. 1697-1716, 1986.

[3] M. Y. Khaled, D. E. Beskos, and E. C. Aifantis, "On the theory of consolidation with double porosity-III A finite element formulation," International Journal for Numerical and Analytical Methods in Geomechanics, vol. 8, no. 2, pp. 101-123, 1984.

[4] G. I. Barenblatt, I. P. Zheltov, and I. N. Kochina, "Basic concept in the theory of seepage of homogeneous liquids in fissured rocks (strata)," Journal of Applied Mathematics and Mechanics, vol. 24, no. 5, pp. 1286-1303, 1960.

[5] M. A. Biot, "General theory of three-dimensional consolidation," Journal of Applied Physics, vol. 12, no. 2, pp. 155-164, 1941.

[6] R. de Boer, Theory of Porous Media, Highlights in the Historical Development and Current State, Springer, Berlin, Germany, 2000.

[7] N. Khalili and S. Valliappan, "Unified theory of flow and deformation in double porous media," European Journal of Mechanics-A/Solids, vol. 15, no. 2, pp. 321-336, 1996.

[8] N. Khalili and A. P. S. Selvadurai, "On the constitutive modelling of thermo-hydro-mechanical coupling in elastic media with double porosity," Elsevier Geo-Engineering Book Series, vol. 2, pp. 559-564, 2004.

[9] J. G. Berryman and H. F. Wang, "The elastic coefficients of double-porosity models for fluid transport in jointed rock," Journal of Geophysical Research, vol. 100, no. 12, pp. 24611-24627, 1995.

[10] J. G. Berryman and H. F. Wang, "Elastic wave propagation and attenuation in a double-porosity dual-permeability medium," International Journal of Rock Mechanics and Mining Sciences, vol. 37, no. 1-2, pp. 63-78, 2000.

[11] M. Svanadze, "Fundamental solution in the theory of consolidation with double porosity," Journal of the Mechanical Behavior of Materials, vol. 16, no. 1-2, pp. 123-130, 2005.

[12] I. Tsagareli and M. M. Svanadze, "Explicit solution of the boundary value problems of the theory of elasticity for solids with double porosity," Proceedings in Applied Mathematics and Mechanics, vol. 10, no. 1, pp. 337-338, 2010.

[13] M. Svanadze and S. De Cicco, "Fundamental solutions in the full coupled theory of elasticity for solids with double porosity," Archives of Mechanics, vol. 65, no. 5, pp. 367-390, 2013.

[14] M. Svanadze and A. Scalia, "Mathematical problems in the coupled linear theory of bone poroelasticity," Computers \& Mathematics with Applications, vol. 66, no. 9, pp. 1554-1566, 2013. 
[15] I. Tsagareli and L. Bitsadze, "Explicit solution of one boundary value problem in the full coupled theory of elasticity for solids with double porosity," Acta Mechanica, vol. 226, no. 5, pp. 14091418, 2015.

[16] E. Cosserat and F. Cosserat, Theorie des Corps Deformables, Hermann, Paris, France, 1909.

[17] C. Truesdell and R. A. Toupin, "The classical field theories," in Handbuch der Physik, S. Flügge, Ed., vol. 1-3, Springer, Berlin, Germany, 1960.

[18] G. Grioli, Elasticita Asimetria, vol. 50 of Annali di Matematica Pura ed Applicata, 1960.

[19] E. V. Kuvshinskii and E. L. Aero, "Continuum theory of asymmetric elasticity. Equilibrium of an isotropic body," Fizika Tverdogo Tela, vol. 6, no. 9, pp. 2689-2699, 1964 (Russian).

[20] R. D. Mindlin, "Influence of couple-stresses on stress concentrations," Experimental Mechanics, vol. 3, no. 1, pp. 1-7, 1963.

[21] V. A. Palmov, "Fundamental equations of the theory of asymmetric elasticity," Journal of Applied Mathematics and Mechanics, vol. 28, no. 3, pp. 496-505, 1964 (Russian).

[22] A. C. Eringen and E. S. Suhubi, "Nonlinear theory of simple micro-elastic solids-I," International Journal of Engineering Science, vol. 2, no. 2, pp. 189-203, 1964.

[23] W. Novacki, "Couple stresses in the theoty of thermoelasticity," Bulletin of the Polish Academy of Sciences Technical Sciences, vol. 14, article 8, 1966.

[24] A. E. Green and P. M. Naghdi, "The linear theory of an elastic Cosserat plate," Mathematical Proceedings of the Cambridge Philosophical Society, vol. 63, no. 2, pp. 537-550, 1967.

[25] W. Novacki, "On the completeness of stress functions in asymmetric elasticity," Bulletin of the Polish Academy of Sciences: Technical Sciences, vol. 14, no. 7, 1968.

[26] V. D. Kupradze, T. G. Gegelia, M. O. Basheleishvili, and T. V. Burchuladze, Three-Dimensional Problems of the Mathematical Theory of Elasticity and Thermoelasticity, North-Holland Publishing, Amsterdam, The Netherlands, 1979.

[27] W. Novacki, Theory of Asymmetric Elasticity, Polish Scientific, Warszawa, Poland, 1986.

[28] R. D. Gauthier and W. E. Jahsman, "A quest for micropolar elastic constants," Journal of Applied Mechanics, vol. 42, no. 2, pp. 369-374, 1975.

[29] É. A. Bulanov, "On the moment theory of elasticity. Plane deformation. Part 3," Strength of Materials, vol. 30, no. 5, pp. 516-521, 1998.

[30] N. Khomasuridze, "Some problems of thermoelastic equilibrium of a rectangular parallelepiped in terms of asymmetric elasticity," Georgian Mathematical Journal, vol. 8, no. 4, pp. 767784, 2001.

[31] M. A. Kulesh, V. P. Matveenko, and I. N. Shardakov, "Construction and analysis of exact analytical solution of kirsch problem within the cosserat continuum and pseudo-continuum," Journal of Applied Mechanics and Technical Physics, vol. 42, no. 4, pp. 145-154, 2001 (Russian).

[32] E. Providas and M. A. Kattis, "Finite element method in plane Cosserat elasticity," Computers and Structures, vol. 80, no. 27-30, pp. 2059-2069, 2002.

[33] M. A. Kulesh, V. P. Matveenko, and I. N. Shardakov, "Parametric analysis of analytical solutions to one- and two-dimensional problems in couple-stress theory of elasticity," Zeitschrift für Angewandte Mathematik und Mechanik, vol. 83, no. 4, pp. 238248, 2003.
[34] R. Janjgava, "The approximate solution of some plane boundary value problems of the moment theory of elasticity," Advances in Mathematical Physics, vol. 2016, Article ID 3845362, 12 pages, 2016.

[35] N. I. Muskhelishvili, Some Basic Problems of the Mathematical Theory of Elasticity, Noordhoff, Groningen, The Netherlands, 1953.

[36] I. N. Vekua, Shell Theory: General Methods of Construction, Pitman Advanced Publishing Program, Boston, Mass, USA, 1985.

[37] T. V. Meunargia, Development of a Method of I. N. Vekua for Problems of the Three-Dimensional Moment Theory Elasticity, TSU Tbilisi, 1987 (Russian).

[38] R. Janjgava, "Derivation of a two-dimensional equation for shallow shells by means of the method of I. Vekua in the case of linear theory of elastic mixtures," Journal of Mathematical Sciences, vol. 157, no. 1, pp. 70-78, 2009.

[39] R. Janjgava and M. Narmania, "The solution of some twodimensional problems of thermoelasticity taking into account the microtemperature," Journal of Thermal Stresses, vol. 39, no. 1, pp. 57-64, 2016. 


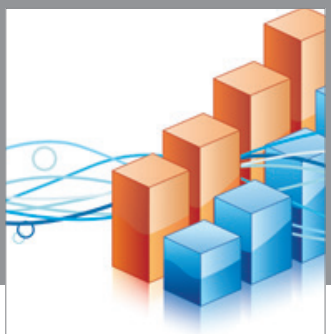

Advances in

Operations Research

vatem alat4

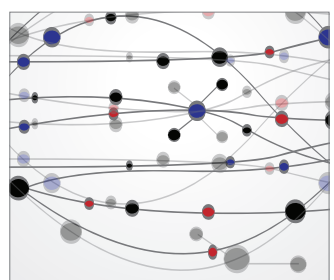

\section{The Scientific} World Journal
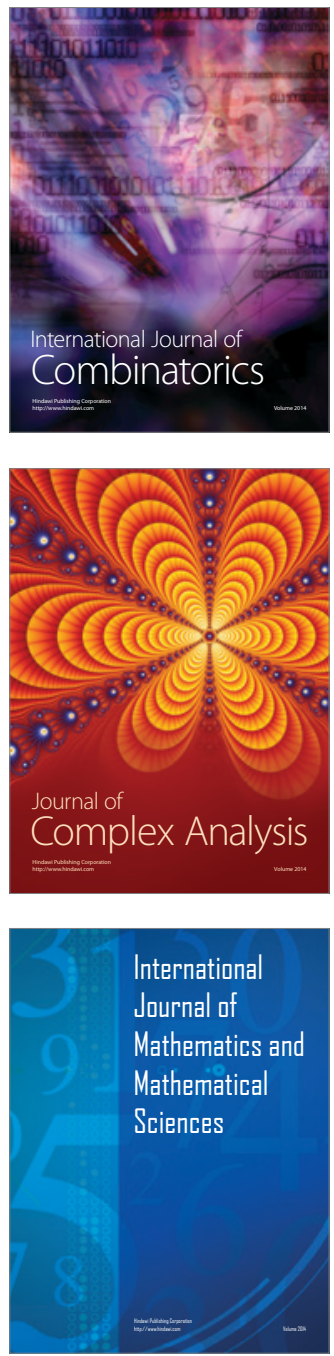
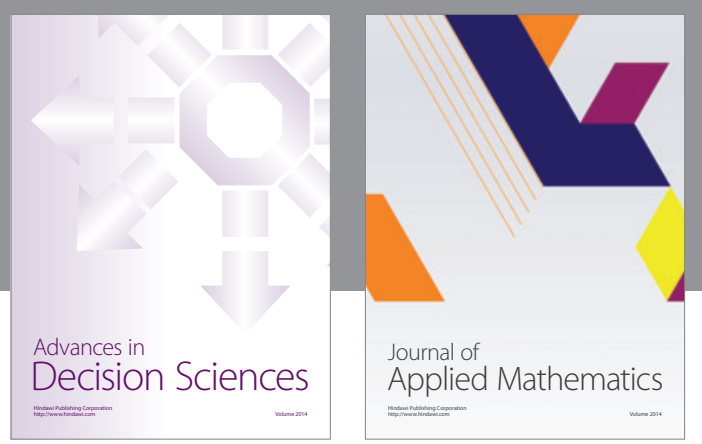

Algebra

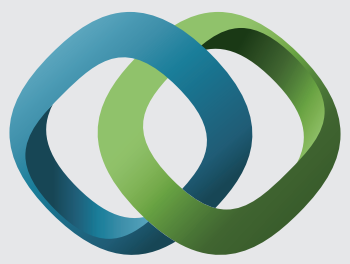

\section{Hindawi}

Submit your manuscripts at

http://www.hindawi.com
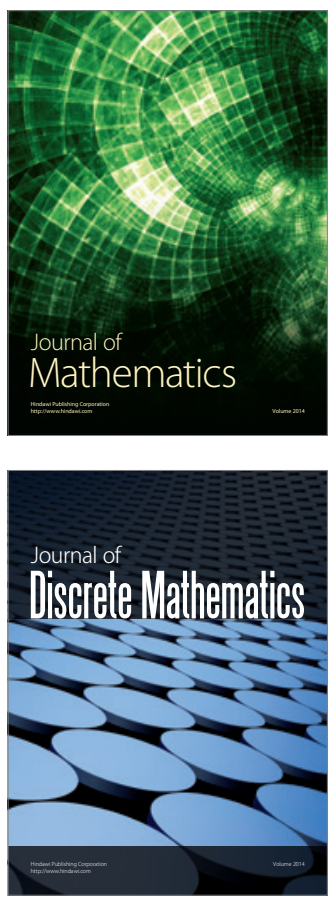

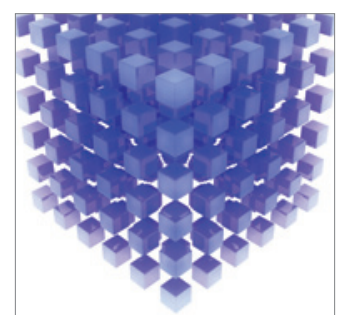

Mathematical Problems in Engineering
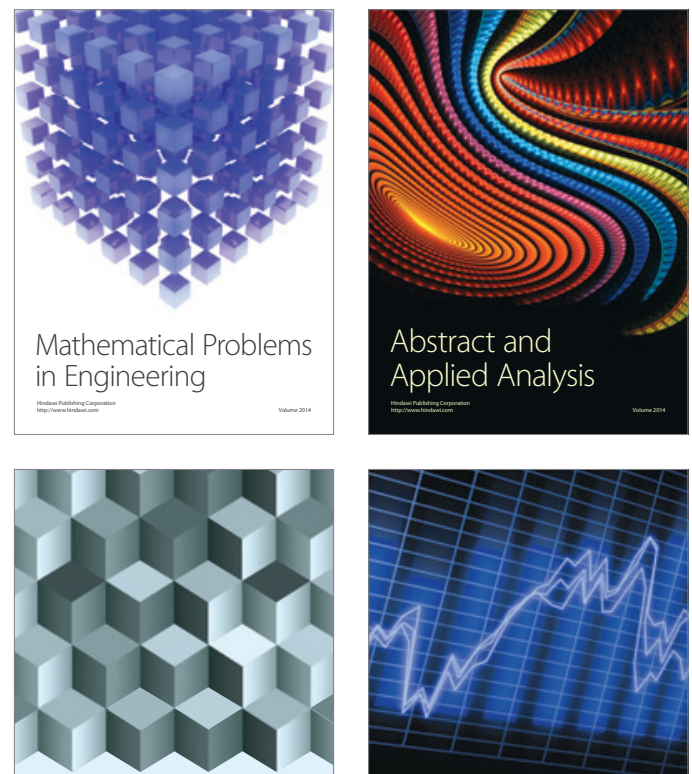

Journal of

Function Spaces

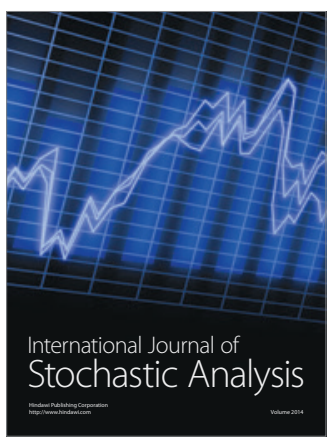

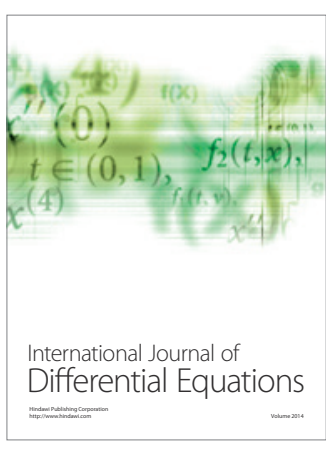
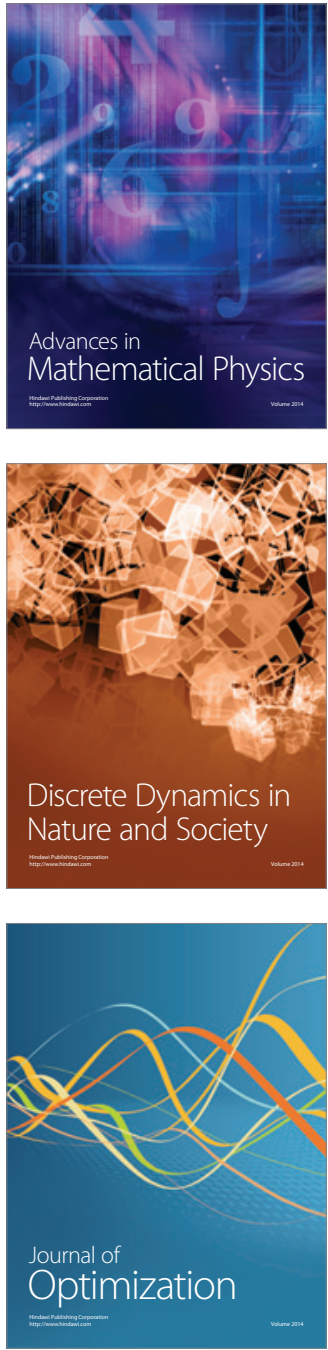